\title{
Surface accuracy achieved by upsetting of preforms generated by laser rod end melting
}

\author{
Heiko Brüning ${ }^{1, a}$ and Frank Vollertsen ${ }^{2}$ \\ ${ }^{1}$ BIAS - Bremer Institut für angewandte Strahltechnik GmbH, Klagenfurterstr. 2, 28359 Bremen, \\ Germany \\ ${ }^{2}$ BIAS - Bremer Institut für angewandte Strahltechnik GmbH, Klagenfurterstr. 2, 28359 Bremen, \\ Germany and University of Bremen
}

\begin{abstract}
Cold forming generally allows the reproduction of parts with a high production rate and low waste of material. For this reason, many small parts for example of the body of a smartphone undergo at least one forming operation during their fabrication, e.g. screws, brackets or springs. However, when the size of forming products is reduced to the micro range, size effects appear and affect the forming process. One size effect leads to the fact that conventional multi stage upsetting processes cannot be scaled to micro range accordingly. As a consequence, the maximum achievable upset ratio decreases from 2.3 in macro range to values below 2 in micro range. This reduces the efficiency of the upsetting process significantly as more upsetting stages have to be carried out to reach the desired shape of the forming product. A very promising approach to reduce required forming stages is the laser rod end melting process which takes advantage of a size effect. Herein, the lower end of a rod is melted by the energy of a laser beam. The molten part of the rod automatically forms spherical due to surface tension. This droplet-shaped part is called "preform". After total cooling, the preform is upset within a single stage cold forming operation to achieve the desired shape. In this paper, results of the upsetting operation with regard to surface accuracy of the forming product are presented. It is shown that surface characteristics with dimensions as small as $500 \mathrm{~nm}$ can be reasonably reproduced.
\end{abstract}

\section{Introduction}

Technical devices such as tablets and smartphones have gained more relevance in daily life during the last few years because their functional abilities are increasing rapidly, thus the influence of metallic micro parts has also been increasing, as predicted by [1]. This trend can easily be visualized by the amount of Apple iPhones sold per year: in 2008, approximately 11 million iPhones were sold worldwide. This value increased to $\sim 170$ mio. iPhones sold in 2014 [2]. Tablets and smartphones as well as laptops consist of many metallic micro parts, which are defined as parts with outer dimensions not exceeding the length of $1 \mathrm{~mm}$ in at least two directions [3], such as screws or springs. As batch sizes for micro parts exceed easily 1 mio. parts per year, production costs and -time is of great relevance.

\footnotetext{
${ }^{\text {a }}$ Corresponding author: bruening@bias .de
}

This is an Open Access article distributed under the terms of the Creative Commons Attribution License 4.0, which permits unrestricted use, distribution, and reproduction in any medium, provided the original work is properly cited. 


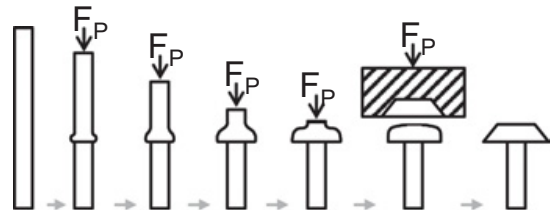

a)



b)

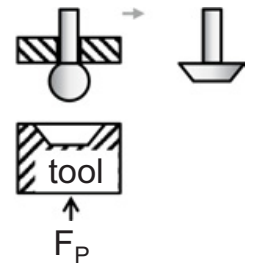

BIAS ID 150499

Figure 1. a) Conventional multi stage upsetting process. b) Laser rod end melting.

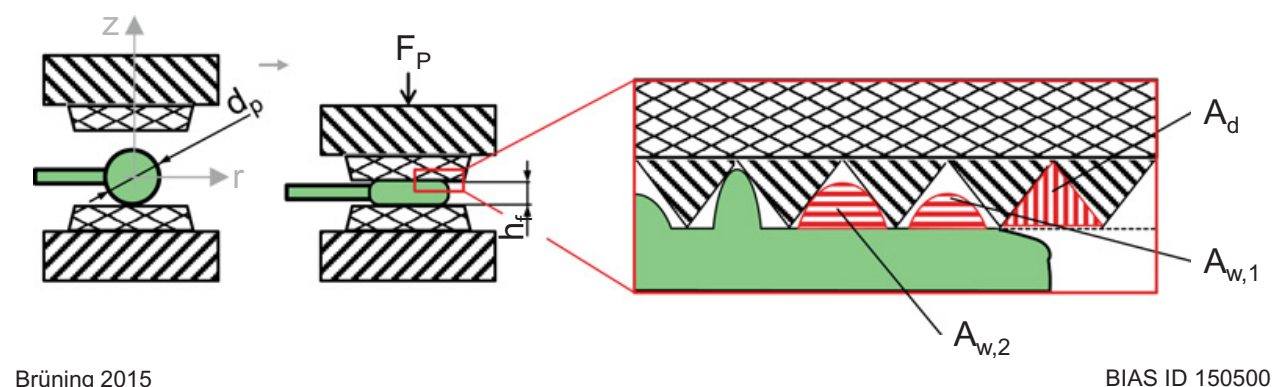

Figure 2. Open die upsetting of preforms to characterize form filling ratio. The enlarged area shows schematically the surface structure and the areas $A_{d}$ and $A_{w}$.

Manufacturing metallic parts by cold forming operations has several advantages compared to other manufacturing process: Cold forming generally allows a high production rate as well as low waste of material [4]. However, when the size of body decreases to micro range, size effects appear [5], which affect the manufacturing process. Because of size effects, the maximum achievable upset ratio decreases to values below 2 compared to 2.3 in macro range [6]. This makes the conventional upsetting process inefficient in micro range as more upsetting stages are required inducing higher production costs (Fig. 1a).

The laser rod end melting process is a new process [7], which allows upset ratios $\gg 200$ within a single process stage [8] and is therefore a good basis for a subsequent forming operation. The effect of increasing influence of surface tension compared to gravitational force in micro range enables the laser rod end melting process wherein the end of a cylindrical rod is melted by a laser beam (Fig. 1b). The melt forms spherical due to surface tension and is called "preform", indicating that a subsequent forming operation is applied to bring the preform in its final geometry, then called the "forming product". It is of interest, to what degree the geometric features of the die are replicated by the geometry of the forming product. Investigations concerning this matter have been carried out by [9] using an alternative sheet metal forming process supported by ultrasonic vibration of the punch. It was found that cavities with dimensions of up to $\sim 700 \mu \mathrm{m}$ could be replicated almost ideally while replication of smaller features with heights $<10 \mu \mathrm{m}$ were found to be more difficult to fill.

In this paper, the ability of the preform to reproduce the surface topography of the die by a bulk metal forming process is investigated. Herein, surface features with sizes ranging from $0.5 \mu \mathrm{m}$ to $6 \mu \mathrm{m}$ are taken into account. 


\section{ICNFT 2015}

Table 1. Parameters of the laser system and process parameters for master forming stage of laser rod end melting.

\begin{tabular}{llllll}
\hline laser system & & & process parameters & \\
\cline { 1 - 2 } \cline { 5 - 5 } laser type & Trumpf TruFiber 300 & & rod diameter & $0.2 \mathrm{~mm}$ \\
\hline wave length & $1085 \mathrm{~nm}$ & & material & AISI 304 (X5CrNi18-10) \\
beam radius & $25.5 \mu \mathrm{m}$ & & laser power, pulse time & $103 \mathrm{~W}, 14 \mathrm{~ms}$ \\
Rayleigh-length & $1.44 \mathrm{~mm}$ & & deflection velocity & $71.9 \mathrm{~mm} / \mathrm{s}$ \\
& & & preform diameter & $0.44 \mathrm{~mm}$ \\
\hline
\end{tabular}

\section{Method to determine form filling}

The preforms are generated by laser rod end melting as indicated by Fig. 1b. To investigate the ability of surface reproduction by cold forming of preforms, the preforms are upset in open dies with parallel surfaces. The shaft of the preform is oriented parallel to the die surface (Fig. 2). After upsetting operation, the average natural strain $\varphi^{*}$ is determined by the initial diameter and final height of the preform:

$$
\varphi^{*}=\ln \left(d_{p} / h_{f}\right)
$$

The average compression stress $\sigma_{C}$ acting on the contact surface $A_{C}$ for a given punch force $F_{P}$ is:

$$
\sigma_{C}=F_{P} / A_{C} .
$$

It is assumed that contact between work piece and die occurs on the whole flattened area of the forming product that is formed during forming operation, well knowing that this assumption might be a simplification (at least for very small values of average compression stress) according to work carried out by [10] using "real contact area" (RCA) which also takes the micro surface structure into account. The RCA can be smaller than $A_{C}$ as some micro grooves exist on both, work piece and tool surface, where at those points direct material contact is not given.

The surface of the die consists of small micro grooves which may exist either on purpose (e.g. as part of a machined hologram) or unintentionally as a consequence of manufacturing process. To characterize the performance of reproduction of micro grooves, the form filling ratio $\Omega$ is defined as follows:

$$
\Omega=\Sigma A_{w} / \Sigma A_{d}
$$

with

$$
\Sigma A_{w}=A_{w, 1}+A_{w, 2}+\ldots+A_{w, n}
$$

similar to the method presented in [9]. In Eq. (3), $\Sigma A_{w}$ is the area of the convex structures of the work piece and $\Sigma A_{d}$ is the area of the according concave structures of the die contour (Fig. 2). It is to be noted that the geometry of the protrusions filling the micro grooves does not have a direct influence on the form filling ratio as only the area $A_{W}$ of each protrusion is taken into account.

Die and forming product are optically surveyed by a confocal 3D laser microscope Keyence VKX200 with a lateral resolution set to $280.3 \mathrm{~nm} /$ pixel and a vertical resolution of $0.1 \mathrm{~nm}$ per measuring plane. The form filling ratio is determined by comparing the contour of the die surface at a certain position relative to the contour of the forming product at the same position to ensure that exactly the correct part of the contact zone is characterized which has been in direct contact during forming operation. For small sections with a length $<4 \mu \mathrm{m}$, an intrusion of $60 \mathrm{~nm}$ of the contour lines is neglected by means of measuring inaccuracy and elastic springback of the preform material. After upsetting of preforms, the surface of the forming product as well as the die is optically surveyed by the 3D-laser microscope to detect the topography. It is assumed that the form filling ratio correlates with acting compression stress as well as with the dimension of the cavity that is supposed to be filled. Therefore, form filling is investigated at two positions of the die with different groove depth and width. 


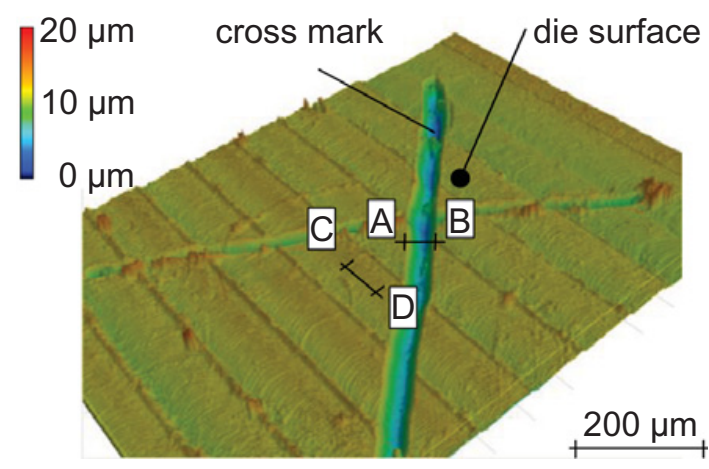

a)

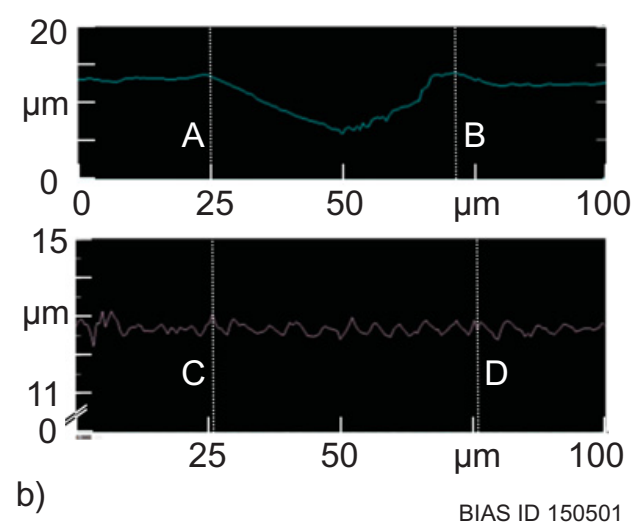

Figure 3. a) Surface texture of the die. b) Contour "AB" and "CD" of the cavities are used for form filling.

\section{Experimental setup}

\subsection{Generation of preforms}

The laser rod end melting process is carried out with a fiber laser TruFiber 300 of Trumpf. A 3D-scan head TurboScan AS30 with a linear translation module LTM20 of Raylase is used. As wrought material, cylindrical AISI 304 (1.4301, X5CrNi18-10) with a diameter of $0.2 \mathrm{~mm}$ is used. The relevant parameters of the master forming stage are in Table 1. The laser beam is focused at the lower end of the rod and deflected according to the movement of the molten preform. Argon is used as shielding gas to prevent oxidation. After total cooling, the preforms are subject to cold forming operation.

\subsection{Upsetting of preforms}

After total cooling, the preforms are upset in a materials testing machine " $250 \mathrm{kN}$ Allround RED" by Zwick. A load cell "X-Force HP $5 \mathrm{kN}$ " is used which allows accuracy class 1 for forces $>10 \mathrm{~N}$. Lower and upper die are of cold work steel AISI D2 (1.2379, X155CrVMo12-1) hardened to 60 HRC. Punch forces between $20 \mathrm{~N}$ and $300 \mathrm{~N}$ are applied to realise average natural strain $\varphi^{*}=0.03 \ldots 0.73$. The upper die surface is prepared with a geometrical mark, similar to a cross, in order to orientate measuring results of the surface topography of the forming product relative to the die. This allows that the according contour measurements of work piece and die are well comparable.

Form filling behaviour is investigated at two positions of the die according to Fig. 3a. At position "AB" a groove with a depth of $6 \mu \mathrm{m}$ and a width of $47 \mu \mathrm{m}$ exists while the milling structure due to fabrication process is very regular at position "CD" with a depth of $0.5 \mu \mathrm{m}$ and a width of $5 \mu \mathrm{m}$. At "CD" the form filling is investigated along the contour for a length of $50 \mu \mathrm{m}$ representing 10 cavities to reduce the influence of measuring inaccuracy.

\section{Results}

The ability of the dendritic preform material to reproduce the die surface topography is investigated by means of form filling ratio $\Omega$ (Eq. (3)) for two surface feature sizes. The form filling ratio is plotted against the average compression stress $\sigma_{C}$ (Eq. (2)) in Fig. 4. It is found that the form filling ratio increases when the average compression stress $\sigma_{\mathrm{C}}$ increases. For surface textures as small as $6.0 \mu \mathrm{m}$ (contour "AB" Fig. 3), $\sim 100 \%$ form filling ratio is achieved for $\sigma_{\mathrm{C}}>1550 \mathrm{~N} / \mathrm{mm}^{2}$. Smaller surface textures represented by the milling structure with a height of $0.5 \mu \mathrm{m}$ (contour "CD" 

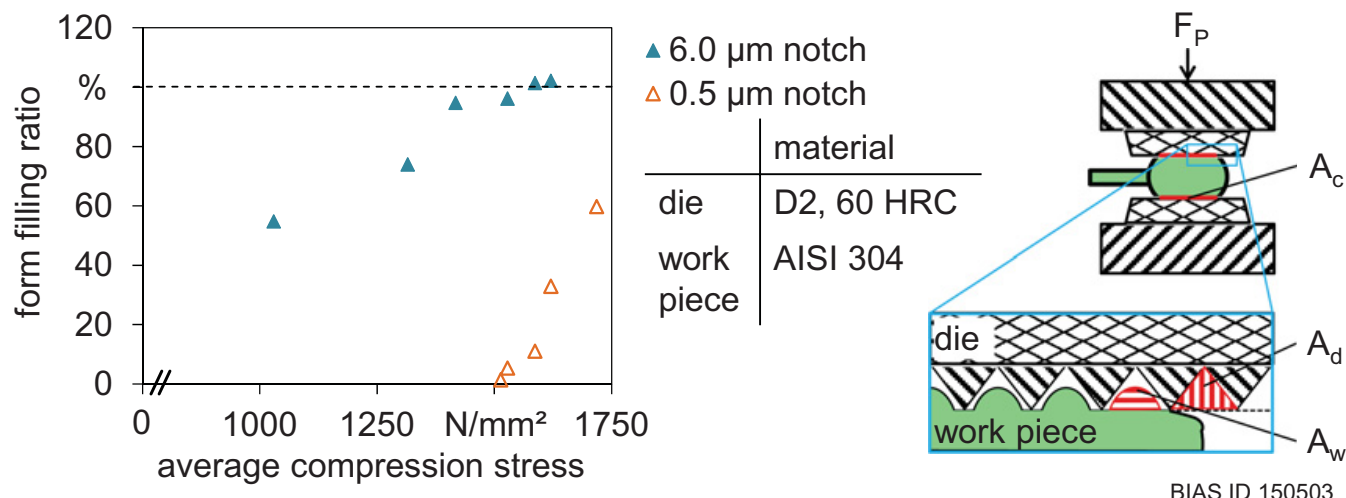

Figure 4. Form filling ratio plotted against average compression stress for upsetting preforms of AISI 304 generated by laser rod end melting.
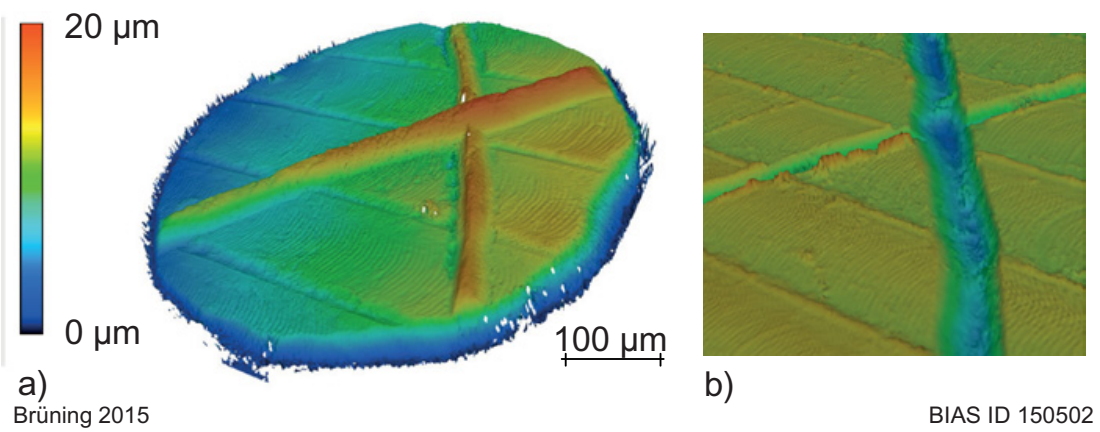

Figure 5. a) Work piece surface after upsetting with $\sigma_{\mathrm{C}}=1720 \mathrm{~N} / \mathrm{mm}^{2}$ leading to a form filling ratio of $\Omega=60 \%$. b) Detail of die surface at the according region.

Fig. 3) cannot be filled to $100 \%$ for the used forming parameters. A maximum of $\Omega=60 \%$ is reached at $\sigma_{\mathrm{C}}=1720 \mathrm{~N} / \mathrm{mm}^{2}$ (Fig. 4). The punch force was not further increased in order to avoid plastic deformation of the small surface features of die. However, it is assumed that the form filling ratio could be further increased if higher contact pressure is realised.

The 3D-measurements from work piece surface and die surface are shown in Fig. 5. The milling structure can be clearly identified on both, die and work piece. According to Eq. (3), a form filling ratio $\Omega=60 \%$ is achieved at this work piece surface.

Figure 6 shows a comparison of both, the die contour due to milling process and the contour of the work piece at the according position that is shown in Fig. 5.

It can be derived that a reasonable reproduction of the die surface is given; the form filling ratio is $\Omega=60 \%$. An intrusion of die surface and work piece can be identified in Fig. 6 for example within a distance of $11 \ldots 15 \mu \mathrm{m}$ of point " $\mathrm{C}$ ". This intrusion can be the result of measuring inaccuracy, either due to uncertainties of measuring positions or due to the limited lateral resolution set to $280.3 \mathrm{~nm} / \mathrm{pixel}$ of the microscope, or it can derive from elastic springback of the work piece material. 



Figure 6. Graph of die and work piece surface by contour measurement of "CD"; punch force $300 \mathrm{~N}, \sigma_{\mathrm{C}}=$ $1720 \mathrm{~N} / \mathrm{mm}^{2}$, average natural strain $\varphi^{*}=0.73$.

\section{Discussion}

A simple method is used to judge the ability of surface reproduction by the so called "form filling ratio" neglecting the shape of the protrusions compared to the shape of the cavities. However, within the cross section of the contour, the areas of the cavities are compared to calculate the form filling ratio. Even though the real shape is not considered, the resulting value of form filling ratio gives a good understanding of the quality of surface reproduction as shown in Fig. 5 and Fig. 6 where surface features in the sub-micrometer range are reasonably reproduced. It is assumed that the aspect ratio (height/width) of the surface features also plays an important role concerning form filling. In this paper, the cavities that were contemplated have an aspect ratio of $\sim 0.1$ so that results concerning this matter have not been taken into account. A further aspect which is not addressed is the contact slip between work piece and die that might also affect the form filling ratio. As the measuring length to determine form filling ratio is relatively large compared to the diameter of the forming product, different length of contact slip would have had to be taken into account even within the measuring length. This has not been done due to measuring uncertainties which are assumed to have a stronger influence on the results. For surface features with a size of $6.0 \mu \mathrm{m}$, excellent form filling has been achieved for average compression stress of $\sigma_{\mathrm{C}}=1550 \mathrm{~N} / \mathrm{mm}^{2}$ without plastic deformation of the die. This result shows that the used die material with a hardness of $60 \mathrm{HRC}$ can be well used for micro cold forming experiments at least for contact pressures applied within these investigations. As die materials usually have a maximum tolerable contact pressure before plastic deformation starts, the die material can be the limiting factor for reproduction accuracy or maximum achievable form filling ratio.

\section{Conclusion}

The laser rod end melting process is used to fabricate preforms. The surfaces of the preforms are calibrated within a single stage cold forming operation. It is found that:

- excellent form filling ratio of $\sim 100 \%$ is achieved for contour heights of $6 \mu \mathrm{m}$ if the average compression stress is $>1550 \mathrm{~N} / \mathrm{mm}^{2}$

- surface structures of $0.5 \mu \mathrm{m}$ height can be reasonable reproduced with form filling ratio of $60 \%$ at an average compression stress of $1720 \mathrm{~N} / \mathrm{mm}^{2}$.

The authors gratefully acknowledge the financial support by Deutsche Forschungsgesellschaft (DFG, German Research Foundation) for subproject A3 "Stoffanhäufen" within the SFB 747 (Collaborative Research Centre) "Mikrokaltumformen - Prozesse, Charakterisierung, Optimierung". 


\section{ICNFT 2015}

\section{References}

[1] M. Geiger, M. Kleiner, R. Eckstein, N. Tiesler, U. Engel, Annals CIRP 50(2), 445 (2001)

[2] www.apple.com, gathered information from Apple Reports Quarter Results (access 19.12.2014)

[3] T. Masuzawa, Annals CIRP 49(2), 473 (2000)

[4] K. Lange, Handbook of Metal forming, ISBN 978-0872634572 (2006)

[5] F. Vollertsen, D. Biermann, H.N. Hansen, I.S. Jawahir, K. Kuzman, Annals CIRP 58(2), 566 (2009)

[6] A. Meßner, Kaltmassivumformung metallischer Kleinstteile: Werkstoffverhalten, Wirkflächenreibung, Prozessauslegung, ISBN 978-3875251005 (1998)

[7] F. Vollertsen, R. Walther, Annals CIRP 57(1), 291 (2008)

[8] A. Stephen, F. Vollertsen, Steel Research International, 220 (2011)

[9] F. Luo, K. Li, J. Zhong et al. J. of Mat. Proc. Tech. 216, 10 (2015)

[10] M. Geiger, U. Engel, F. Vollertsen, Annals CIRP 41(1), 255 (1992) 